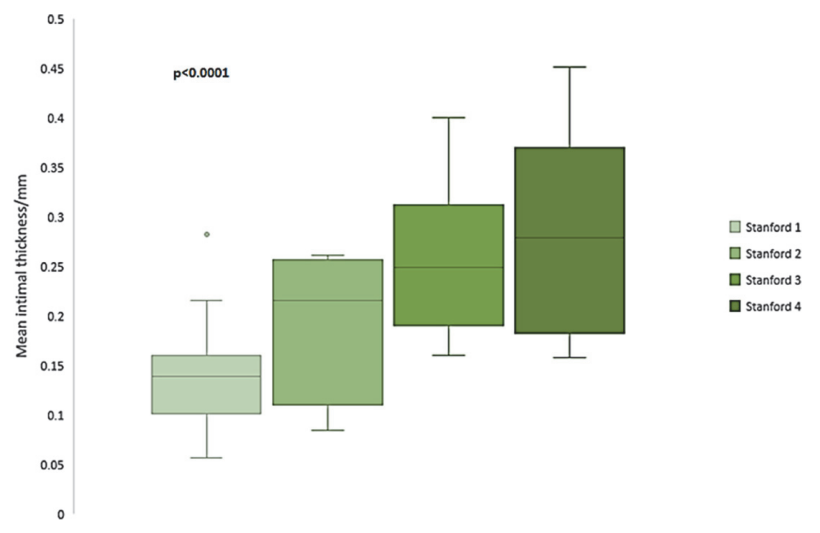

Abstract 123 Figure 1 Box plot showing optical coherence tomography-derived mean intimal thickness of each coronary vessel as a function of intravascular ultrasound-derived Stanford classification. P value relates to the significance of the concordance

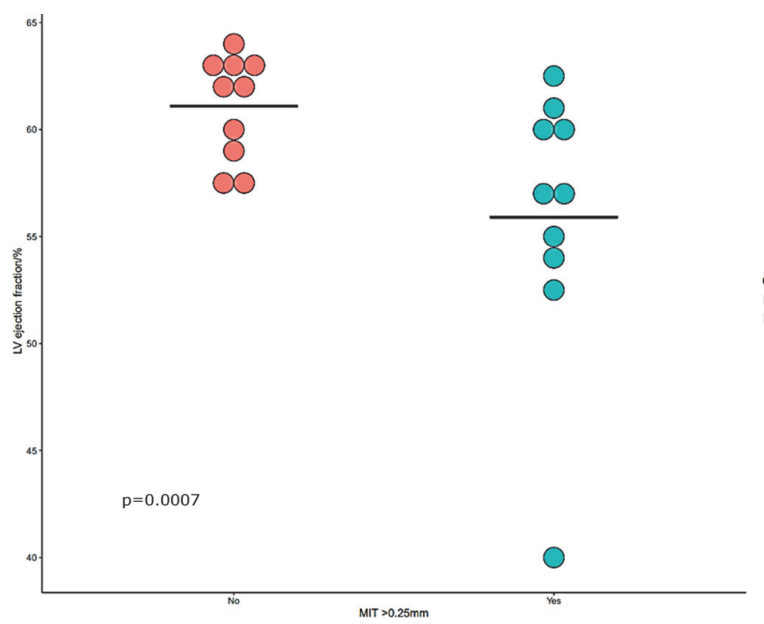

Abstract 123 Figure 2 Dot plot showing left ventricular ejection fraction at time of angiography for patients with and without optical coherence tomography-defined coronary allograft vasculopathy (CAV), defined as mean intimai thickness (MIT) $>0.25 \mathrm{~mm}$. Crossbar represents mean ejection fraction

significantly smaller on OCT: mean intimal thickness (IT) by OCT was $0.21 \pm 0.1 \mathrm{~mm}$ vs $0.44 \pm 0.24 \mathrm{~mm}$ by IVUS, $\mathrm{p}$ $<0.001$. A mean ITOCT $>0.25 \mathrm{~mm}$ had a sensitivity of $86.7 \%$ and specificity of $74.3 \%$ at detecting Stanford grade 4 CAV. Those with CAV evident on ICA had significant reduction in graft ejection fraction (EF) over median follow up of 7.3 years (mean $\triangle \mathrm{EF}-3.6 \%$ with $\mathrm{CAV}$ vs $+3.8 \%$ without CAV, $p=0.04)$. Patients with mean ITOCT $>0.25 \mathrm{~mm}$ in at least one vessel had a lower $\mathrm{EF}$ at time of surveillance $(55.9 \%$ vs $61.1 \%, \mathrm{p}=0.0007)$ (figure 2). Only two MACEs were noted.

Conclusion Coronary imaging with OCT correlates well with IVUS for detection of CAV. Mean IT of $>0.25 \mathrm{~mm}$ on OCT detects Stanford grade 4 CAV with reasonable accuracy and may be a useful cut-point for clinical use.Combined angiography and OCT to screen for CAV within 12-24 months of transplant predicts concurrent and future deterioration in left ventricular function, thus may trigger early alterations to clinical management to prevent clinical worsening.

Conflict of Interest none

\section{COMPARING THE SAFETY AND FEASIBILITY OF IMPLANTING PULMONARY ARTERY PRESSURE MONITORS VIA THE INTERNAL JUGULAR VEIN COMPARED TO STANDARD FEMORAL VENOUS ACCESS IN PATIENTS WITH PULMONARY ARTERIAL HYPERTENSION}

Jennifer Middleton, Hamza Zafar, David.G. Kiely, Alexander Rothman. University of Sheffield, Sheffield, UK

\subsection{6/heartjnl-2021-BCS.121}

Aim To review feasibility and safety of implanting pulmonary artery pressure (PAP) monitors via the right femoral vein (RFV) versus the right internal jugular vein (RIJV) in a cohort of patients with pulmonary arterial hypertension (PAH) referred to the National PH centre in Sheffield.

Background The implantation of PAP monitors is currently licenced via the RFV during a standard right heart catheterisation (RHC). Although access via RFV and RIJV for RHC have been shown to be safe, procedures undertaken via the RIJV can be quicker, better tolerated and shown to have a significantly lower risk of complication than those via the RFV, and can reduce length of stay by facilitating same day discharge.

Methods From January 2020 to March 2021, 15 PAP monitors were implanted in patients with WHO-FC III PAH and a hospital admission within the past year as part of the FIT-PH study (Feasibility of novel clinical Trial infrastructure, design and technology for early phase studies in patients with Pulmonary Hypertension) (19/YH/0354). Catheter lab reports, radiology records and clinical records were reviewed to compare safety and screening/procedure time and radiation dose of RFV and RIJV access.. Implants were performed by an interventional-trained consultant cardiologist with experience in heart failure and $\mathrm{PH}$.

Results 8 implants were undertaken via RFV access and 7 via RIJV. Demographics and background therapy were well matched between groups. No procedure or device-related complications were identified. Screening time for RFV procedures: 22 mins 5 secs \pm 13.2 compared to RIJV procedures: 8 mins \pm 4 ( $\mathrm{p}$ value 0.0155 ) and RFV radiation dose: 22.5 GYCM2 \pm 20.6 compared to RIJV: 18.5 GYCM2 \pm 16 ( $p$ value NS).DiscussionPatients with PAP sensors implanted via the RIJV had a significantly reduced screening time and a reduced radiation dose although this was non-significant. RIJV procedures were well tolerated and quicker procedures. RIJV implantation also facilitated same day discharge potentially reducing costs.

Conclusion The implantation of PAP monitors via the RIJV in patients with patients was found to be a safe and feasible alternative to RFV access in patients with PAH. Significantly reduced procedure times with RIJV procedures facilitated early and safe discharge.

Conflict of Interest none

\section{CARDIAC METABOLIC FLEXIBILITY AND MYOCARDIAL SUBSTRATE UTILISATION IN RESPONSE TO PHARMACOLOGICAL STRESS IN TYPE 2 DIABETES}

'Lavanya Athithan, ${ }^{1}$ Kelly S Parke, ${ }^{2}$ Andrew Ladwiniec, 'David Adlam, ${ }^{1}$ Gaurav S Gulsin, ${ }^{1}$ Manjit Sian, ${ }^{1}$ Anna-Marie Marsh, 'Gerry P McCann, ${ }^{3}$ Eylem Levelt. ${ }^{1} \mathrm{NIHR}$ Leicester Biomedical Research Centre, University of Leicester, Leicester, UK; ${ }^{2}$ University Hospitals of Leicester NHS Trust; ${ }^{3}$ University of Leeds, Multidisciplinary Cardiovascular Research Centre and Biomedical Imaging Science

10.1136/heartjnl-2021-BCS.122 
Background Efficient matching of energy supply to demand is essential for maintaining normal cardiac function. Altered cardiac metabolism may contribute to the development of cardiac dysfunction by impairing metabolic flexibility in type 2 diabetes (T2D). Utilising simultaneous coronary sinus (CS) and aorta blood sampling and cardiac magnetic resonance imaging (CMR), we aimed to evaluate the effect of T2D on myocardial substrate preferences in response to acute increases in cardiac workload and the effects on contractile function.

Methods Eligible participants without obstructive coronary artery disease $(>50 \%$ luminal coronary artery stenosis on coronary angiography) underwent transmyocardial arteriovenous blood sampling. Metabolites in paired coronary sinus and arterial samples were quantified to determine myocardial fuel selection at rest and during a stress protocol with intravenous dobutamine. Fatty acid (FA), glucose, 3-hydroxybutyric acid (3HBA) and lactate utilisation at rest and haemodynamic stress was calculated as an extraction fraction \% (EF). Participants underwent dobutamine stress multiparametric CMR imaging at 3.0 Tesla (Skyra, Siemens, Germany) on a separate visit within 21 days to quantify cardiac volumes, function and perfusion.

Results Two thousand and sixty-one participants were screened and due to stringent inclusion and exclusion criteria for this mechanistic study, three T2D patients and five matching controls were enrolled. Baseline demographics and glycometabolic

Abstract 125 Table 1 Baseline demographics and glycometabolic data

\begin{tabular}{lll}
\hline & T2D $(\mathbf{n}=3)$ & Controls $(\mathrm{n}=5)$ \\
\hline Age (yr) & $60 \pm 2$ & $59 \pm 15$ \\
Gender (M) & 3 & 4 \\
BMI (kg/m2) & $29.8 \pm 7.1$ & $31.3 \pm 5.8$ \\
Weight (kg) & $85.4 \pm 24.3$ & $91.0 \pm 22.9$ \\
Systolic BP (mmHg) & $121 \pm 7$ & $129 \pm 10$ \\
Diastolic BP (mmHg) & $78 \pm 2$ & $79 \pm 14$ \\
Baseline Heart Rate (bpm) & $57 \pm 10$ & $62 \pm 5$ \\
Peak Stress Heart Rate (bpm) & $120 \pm 7$ & 11614 \\
Fasting glucose (mmol/L) & $7.1 \pm 2.4$ & $5.1 \pm 0.6$ \\
HOMA IR & $14.1 \pm 9.7$ & $3.9 \pm 1.6$ \\
Total Cholesterol: HDL & $3.0 \pm 0.2$ & $3.0 \pm 0.8$ \\
HbA1C (mmol/mol) & $52 \pm 19$ & $37 \pm 2$ \\
\hline
\end{tabular}

Abstract 125 Table 2 Echocardiographic and CMR Data

\begin{tabular}{lll}
\hline & T2D $(n=3)$ & Controls $(n=5)$ \\
\hline Echocardiographic & & \\
Data & & \\
E/A & $0.99 \pm 0.28$ & $0.92 \pm 0.20$ \\
Average E/E' & $15.17 \pm 7.73$ & $8.50 \pm 3.84$ \\
CMR Data & & \\
LV EDVi (ml/m2) & $101.5 \pm 43.8$ & $82.6 \pm 8.9$ \\
LVEF (\%) & $53.0 \pm 21.7$ & $59.9 \pm 4.1$ \\
LV Mass Index (g/ & $66.0 \pm 23.0$ & $63.7 \pm 13.0$ \\
m2) & & \\
LV Mass/Nolume & $0.66 \pm 0.05$ & $0.76 \pm 0.09$ \\
(g/ml) & & \\
\hline
\end{tabular}

LV EDV: Left Ventricular End Diastolic Volume Index; LVEF: Left Ventricular Ejection
Mean Extraction Fraction of 3 Hydroxybutyric Acid (3-HBA) Rest vs Stress

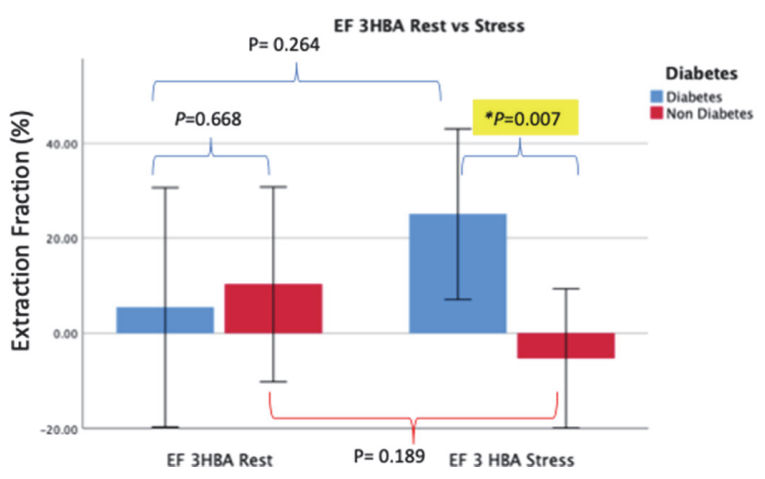

Abstract 125 Figure 1

results are documented in table 1. Mean 3-HBA changes calculated as extraction fraction at stress and rest are illustrated in figure 1. Table 2 shows the major echocardiographic and CMR results. There were no significant differences in FA or glucose uptake between T2D patients and controls at rest or stress. 3HBA EF was significantly increased in T2D during stress $(25.04 \%$ vs $-5.31 \%, \mathrm{p}=0.007)$.

Conclusions We demonstrate for the first time in vivo that the diabetic heart switches to ketone bodies during increased workloads as a significant fuel source. At present, it is unknown whether enhanced ketone body metabolism in T2D is beneficial, maladaptive, or a bystander. As the energetic properties of ketones are favourable, increased myocardial ketone oxidation could be an adaptive change designed to compensate for defects in myocardial energy metabolism in diabetes. Further, larger studies are warranted.Funding acknowledgement: This research was funded by the British Heart Foundation Clinical Research Training Fellowship and the Wellcome Trust on a Seed Grant

Conflict of Interest None

\section{IS HEART FAILURE ADMISSION OVER WEEKENDS AND BANK HOLIDAYS ASSOCIATED WITH HIGHER RISK OF DEATH?}

${ }^{1}$ Reham Awad, ${ }^{1}$ Prince Joseph, 'Emily Owen, 'Shamshad Khan, ${ }^{1}$ Mohammed Hilal-Babu, ${ }^{1}$ Sunita Avinash, 'Sundas Masudi, 'Jameela Bahar, ${ }^{1}$ Haydn Bennett, ${ }^{2}$ Kenneth Wong, ${ }^{1}$ Amna Rahman. 'Blackpool Teaching Hospitals NHS Foundation Trust (Blackpool Victoria Hospital), Blackpool, UK; ${ }^{2}$ Blackpool Teaching Hospitals

\subsection{6/heartjnl-2021-BCS.123}

Introduction - Acute Heart failure inpatient mortality is as high as $7-11 \%$ in UK National Heart Failure audits with little improvement over many years. Some but not all studies have suggested a possible weekend effect on mortality in different patient groups including patients suffering from heart failure. However, some studies failed to recognise admissions after $5 \mathrm{pm}$ on Fridays as weekend. We aim to determine in a large cohort of heart failure admissions whether patients are less likely to survive if admitted during weekend or Bank Holidays.

Methods - A retrospective study of consecutive admissions with heart failure as 1st diagnostic position in Blackpool Victoria Hospital over an 18 month period from 1st August 